\title{
Abnormal Communication between Lateral Thoracic Artery and Anterior Circumflex Humeral Artery - a Case Report
}

\author{
Dinesh V. Kumar*, Ramakrisnan Rajprasath, Prasad G. Bhavani
}

\section{ABSTRACT}

Variations in the branching pattern of axillary artery are observed by many anatomists all over the world. A unique bilateral variation in the axillary artery was observed during the routine dissection of the upper limbs on an approximately 65 year old male cadaver. An abnormal communicative channel was observed between lateral thoracic artery and anterior circumflex humeral artery. It passed between the two roots of median nerve. Arterial anomalies in the upper limb are due to defective remodelling of vascular plexus of the upper limb bud during embryogenesis. Knowledge of variations in axillary artery is quintessential for surgeons, radiologists and anaesthesiologists to avoid treacherous complications during procedures.

\section{KEYWORDS}

axillary artery; abnormal communicative channel; lateral thoracic artery; circumflex humeral artery

\section{AUTHOR AFFILIATIONS}

Department of Anatomy, Pondicherry Institute of Medical Sciences, Puducherry, India

* Corresponding author: Department of Anatomy, Pondicherry Institute of Medical Sciences, Puducherry - 605014, India; e-mail: dinesh.88560@gmail.com

Acta Medica (Hradec Králové) 2018; 61(2): 65-68 https://doi.org/10.14712/18059694.2018.54

(c) 2018 The Authors. This is an open-access article distributed under the terms of the Creative Commons Attribution License (http://creativecommons.org/licenses/by/4.0), which permits unrestricted use, distribution, and reproduction in any medium, provided the original author and source are credited. 


\section{INTRODUCTION}

Axillary artery being the salient blood vessel of the upper limb extends from the outer border of first rib to the lower border of teres major muscle. It is in intimate relationship with the cords of brachial plexus and its branches and enclosed with the axillary sheath. In anatomical position, artery presents a bold convex curve directed upward and laterally. The artery is divided into three parts by pectoralis minor muscle. Three cords of brachial plexus are named according to their relation to the second part of axillary artery.

Superior thoracic artery is the only branch emanating from the first part of axillary artery. It usually anastomoses with internal thoracic and upper intercostal arteries (1). Of the two branches from the second part of axillary artery, thoraco-acromial artery is shorter and divides into acromial, deltoid, clavicular and pectoral branches. Lateral thoracic artery usually arises from the second part of axillary artery and runs along the lateral border of pectoralis minor muscle. It is the major blood supply to the pectoral muscles, subscapularis and serratus anterior. In addition, it supplies the nipple-areolar complex. One of the devastating complications in plastic reconstruction procedures of the breast is necrosis of the nipple-areolar complex which occurs when there is an irreversible lateral thoracic arterial insufficiency (2). Stook et al. (3) classified the arteries arising from the proximal two-thirds of the axillary artery into two classes: "deep arteries" including the lateral thoracic and superior thoracic arteries; and "superficial artery" (thoraco-acromial artery).

Third part of axillary artery gives three branches namely anterior circumflex humeral artery, posterior circumflex humeral artery and subscapular artery. The anterior circumflex humeral artery runs deep to coracobrachialis and both heads of biceps (4). It then participates in anastomoses with posterior circumflex humeral artery. Median nerve which usually lies anterior to axillary artery is formed by the union of medial and lateral roots from medial and lateral cords of brachial plexus respectively. Knowledge of branching pattern in this region is essential for orthopaedic surgeons attempting to reduce old dislocations and fractures (1).

\section{CASE REPORT}

During routine dissection of the axilla, a bilateral variation was found in the typical branching pattern in an approximately 65 year old formalin fixed male cadaver. The axillary artery and its branches were identified after clearing the fat. The first two parts of the axillary artery and its branches were normal. The third part of the axillary artery gave three branches as described. Median nerve was formed by medial and lateral roots coming from medial and lateral cords of brachial plexus respectively as routinely described.

An abnormal arterial trunk (Fig. 1) was given off from the lateral thoracic branch of the second part. It then coursed downwards and laterally (Fig. 2) between the two roots of the median nerve and joined the anterior circum-

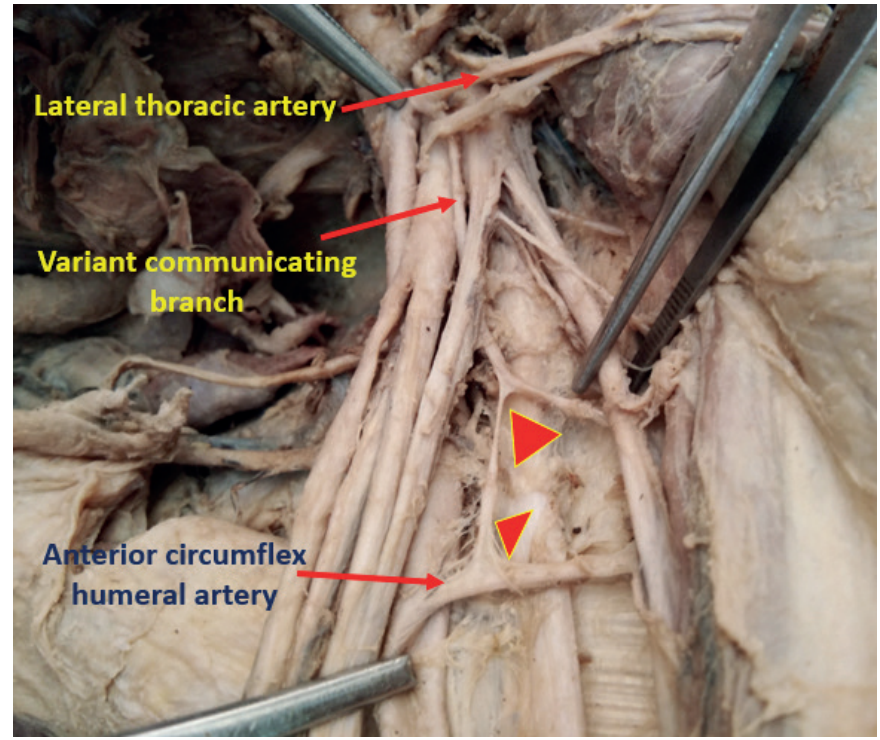

Fig. 1 Dissection of left axilla showing the abnormal communicative vessel (arrow heads) between lateral thoracic artery and anterior circumflex humeral artery. The aberrant vessel passes between the two roots of median nerve.

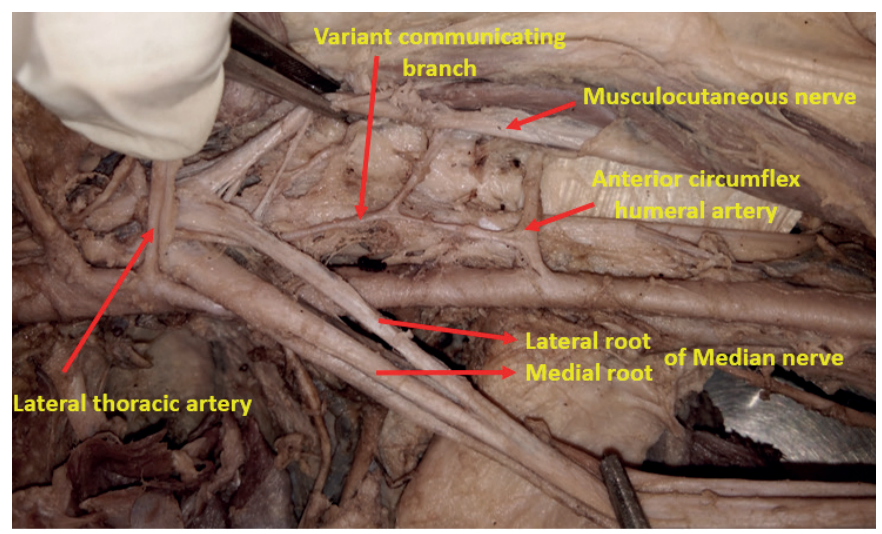

Fig. 2 Course of abnormal communicative channel parallel to axillary artery. A muscular branch can also be noticed emanating from the trunk of aberrant vessel

flex humeral artery of the third part (Fig. 3). The length of the arterial trunk was $5.4 \mathrm{~cm}$ and the diameter was found to be smaller than that of lateral thoracic artery. As the trunk was found between the roots of the median nerve, there are chances for it to get compressed. There were no evident signs of stenosis in subclavian, axillary and brachial arteries, which ruled out the possibility of the abnormal trunk being an anastomotic channel. The distribution of other arteries in the upper limb were found to be normal.

\section{DISCUSSION}

Earlier studies by many anatomists showed that variations in the upper limb vasculature, particularly in the branching pattern of axillary artery are not so uncommon. In a study (5), it was observed that the normal pattern of distribution is seen in $20 \%$ of specimens and the rest 


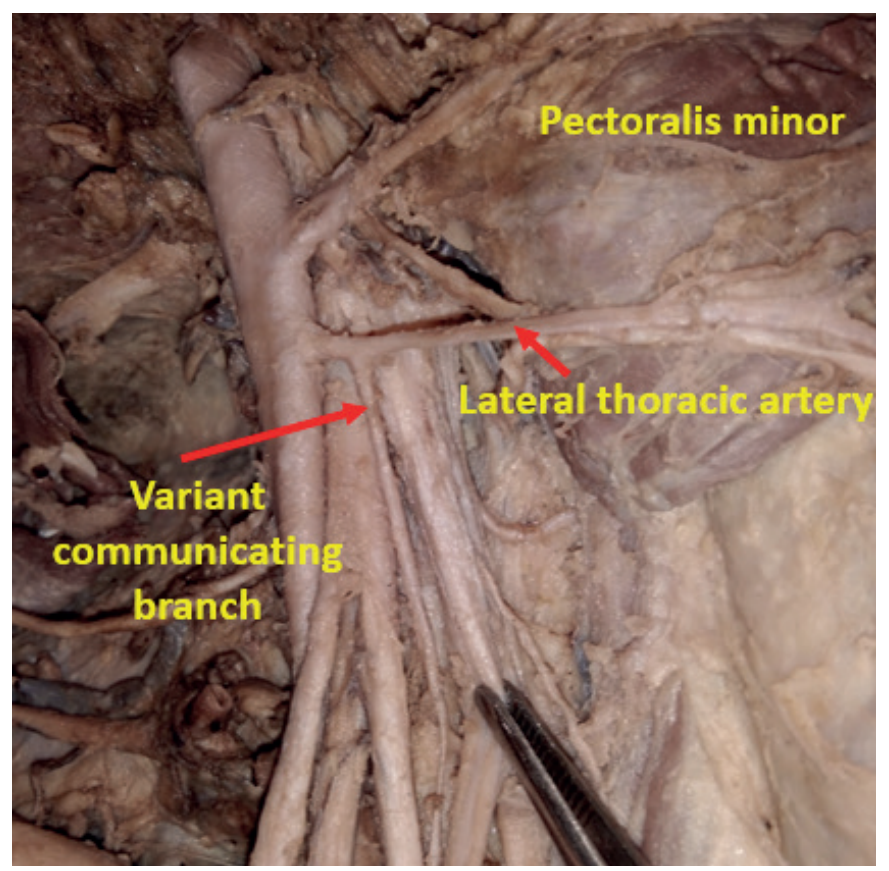

Fig. 3 Proximal portion of aberrant vessel emanating from lateral thoracic artery.

of $80 \%$ specimens showed variations in the origin, branching pattern and distribution. In our case report, abnormal communicative channel between lateral thoracic artery and anterior circumflex was observed on both sides.

Studies $(6,7)$ have documented the common arterial trunk from third part of axillary artery which then divided into branches. Another study (8) documented a common trunk from the second part of axillary artery which then gave origin to thoracoacromial, lateral thoracic, subscapular, and posterior circumflex humeral arteries.

The embryological basis of such abnormal communicative channel might be due to the persistence of communicative channels in the primitive vascular plexus which are usually obliterated during the later stage of embryogenesis (9). Any conditions capable of inducing a regional hypoxia, can also promulgate the formation of such abnormal channels and most of those acquired conditions are mostly unilateral. Any abnormal communication between vessels establishes two circuits in which the blood, flows through two separate systems. One circuit (usually the normal one) characterized by high arterial pressure and high peripheral resistance and the other circuit (abnormal) being shorter and of low arterial pressure.

During the initial phase of embryogenesis, endothelial cells are formed first, and undergo proliferation and coalescence into capillary plexus (10). This capillary plexus then gets remodelled into endothelial tubules and later into blood vessels. Various molecular factors such as VEGFR2 and FGFR1 can maintain endothelial formation (10). Abnormal shear stress can act as an endothelial -inducing mesenchymal transition stimulus, both in development and in adult settings (11). A new vessel formation is triggered by endothelial cell activation and sprouting coordinated with controlled detachment of the surrounding mural cells and sustained by further remodelling of vessel wall (12). Pre-existing vessel might also split by a process known as "intussusception" giving rise to daughter vessels (13). Quiescent endothelial cells, if triggered by suitable molecular factors, get modified into a "tip cell" which then detaches from the basement membrane and initiates the formation of a vascular branch (13). The tip cells migrate under response of molecular factors such as semaphorins and ephrins. Later lumen gets established and forms a new vessel (14).

The primary arterial trunk of the mammalian forelimb develops from seventh intersegmental artery (15). The axillary arterial plexus gets remodelled into longitudinal trunks and with the formation of brachial plexus; they get united to form the adult structure. The initial capillary plexus enters the upper limb bud at stage 12. This plexus begins to differentiate at stage 13 remodelling process starts in the aorta and continues in a proximal to distal sequence. By stage 15 the differentiation has reached the subclavian and axillary arteries (15). Absence of evident signs of arterial stenosis and bilateralism of the variation ensure its congenital aetiology.

The abnormal communicative channel documented in our report, runs between two roots of median nerve. The possible embryological origin for it might be the eighth or ninth intersegmental arteries. This is in concordance with Yang et al. (16), who had postulated with each instance of segmental artery variation also involved a variation in spatial relationship to the branches of brachial plexus. In worst case scenario, the abnormal vessel can get compressed between the roots of median nerve.

Knowledge regarding the variations in axillary vasculature is critical for the surgeons to perform axillary lymph node clearance or excision biopsy in cases of breast carcinoma, in cases of subclavian artery occlusion, aneurysm of axillary artery and axillary haematoma.

Understanding the variations of humeral arteries helps the plastic surgeons to generate flaps for scar releasing procedures of the head and neck (17). Knowing about the variations in humeral arteries is of significance in vascular injuries after proximal humeral fractures. Anatomy of axillary artery is also of utmost importance in cardiothoracic vascular surgery, as they are one of the cannulation sites for bypass procedures (18).

\section{CONCLUSION}

Arterial variations in upper limb are clinically important for the surgeons, anaesthetists, interventional radiologists and orthopaedic surgeons. Here we report an abnormal communicative channel between lateral thoracic artery and anterior circumflex humeral artery. The knowledge of such variations should be disseminated to help the clinicians arriving at an accurate diagnostic interpretation and to plan optimal therapeutic intervention.

\section{CONFLICT OF INTEREST}

The authors hereby declare that they have no conflict of interest. 


\section{REFERENCES}

1. Standring S, editor. Gray's anatomy: the anatomical basis of clinical practice. Forty-first edition. New York: Elsevier Limited, 2016.

2. Van Deventer PV, Page BJ, Graewe FR. The safety of pedicles in breast reduction and mastopexy procedures. Aest Plast Surg 2008; 32: 30712.

3. Stook FP, Zonnevijlle EDH, Groen GJ. A reappraisal of the blood supply of the pectoralis minor muscle. Clin Anat 1994; 7: 1-9.

4. Sinnatamby CS, Last RJ, editors. Last's anatomy: regional and applied. 12th ed. Edinburgh; New York: Churchill Livingstone/Elsevier, 2011.

5. Thampi SM, Vijayamma KN, Kumar RP. Axillary artery - A study on branching pattern and variations. Journal of Evidence Based Medicine and Healthcare 2017; 4(43): 2619-24.

6. Rao RT, Shetty P, Suresh R. Abnormal branching pattern of the axillary artery and its clinical significance. International Journal of Morphology 2008; 26(2): 389-92.

7. Samuel VP, Vollala S, Nayak S, Rao M, Bolla SR, Pammidi N. A rare variation in the branching pattern of the axillary artery. Indian Journal of Plastic Surgery 2006; 39(2): 222-23.

8. Srimathi T. Abnormal branching pattern of the axillary artery-a case report. Int J Basic Med Sci 2011; 2: 73-6.

9. Arey LB. Developmental Anatomy. In: Development of the arteries. 6th Edition. Philadelphia: W. B. Saunders Co, 1957: 375-7.

10. Dejana E, Hirschi KK, Simons M. The molecular basis of endothelial cell plasticity. Nat Commun 2017; 8: 14361.
11. Ten Dijke P, Egorova AD, Goumans MJ, Poelmann RE, Hierck BP. TGF-beta signalling in endothelial-to-mesenchymal transition: the role of shear stress and primary cilia. Sci Signal 2012 Feb 21; 5(212): pt2.

12. Murakami M. Signaling required for blood vessel maintenance: molecular basis and pathological manifestations. International Journal of Vascular Medicine 2012; Article ID 293641.

13. Carmeliet $P$ and Jain RK. Molecular mechanisms and clinical applications of angiogenesis. Nature 2011; 473(7347): 298-307.

14. Adams RH, Eichmann A. Axon guidance molecules in vascular patterning. Cold Spring Harb Perspect Biol 2010; 2: a001875.

15. Rodríguez-Niedenführ M, Burton GJ, Deu J, Sañudo JR. Development of the arterial pattern in the upper limb of staged human embryos: Normal development and anatomic variations. J Anat 2001; 199(4): 407-17.

16. Yang HJ, Gil YC, Lee HY. Intersegmental origin of the axillary artery and accompanying variation in the brachial plexus. Clin Anat 2009; 22: 586-94

17. Halmy C, Szetei K, Na'dai Z, et al. Posterior circumflex humeral artery perforator flap (PCHAP-flap) in axillary scar release. Orv Hetil 2009; 150: 603-6.

18. Bonatti J, Coulson AS. The subclavian and axillary arteries as inflow vessel for coronary artery bypass grafts combined experience from three cardiac surgery centres. Heart Surg Forum 2000; 3: 307-12. 